3. A mark for each face, and a list of the edges and vertices in their order upon the boundary of each face.

Such a notation must contain a mark of distinction for the two sides of an edge; an easy matter if the direction of positive rotation be adopted uniformly in listing arrangements about the vertices and faces respectively.

These processes, and the proved existence of fundamental polygons, open a range of particular problems of considerable interest. But of even superior interest must be, at least until it is solved, the problem of finding a method for constructing, a priori, upon a given surface the exceptional (Davis) special reticulations whose characteristics are given by the restrictive tables.

NORTH WESTERN UNIVERSTTY, April, 1898.

\title{
SYSTEMS OF SIMPLE GROUPS DERIVED FROM THE ORTHOGONAL GROUP.
}

BY DR. L. E. DICKSON.

1. In the February number of the Bulletrs I determined the order $\omega$ of the group $G$ of orthogonal substitutions of determinant unity on $m$ indices in the $G F\left[p^{n}\right]$ and proved that, for $* p^{n}>5, p \neq 2$, the group is generated by the substitutions

$$
O_{i, j}^{\alpha, \beta}: \quad \begin{aligned}
& \xi_{i}^{\prime}=\alpha \xi_{i}+\beta \xi_{j}, \\
& \xi_{j}^{\prime}=-\beta \xi_{i}+\alpha \xi_{j},
\end{aligned} \quad\left(\alpha^{2}+\beta^{2}=1\right) .
$$

The structure of $G$ was determined for the case $p=2$. I have since proved $\dagger$ that for every $m>4$ and every $p^{n}>5$ of the form $8 l+3$ or $8 l+5$, the factors of composition of $G$

* The fact that $p^{n}=3$ is an exception was not pointed out in the BULLETIN. In fact Jordan hall not proven case $2^{\circ}$ of $\& 211$ when $-1=$ square, so that the case $a^{2}=b^{2}=c^{2}=\ldots=1$ was unsolved when $p=3$, $m=3 k+1$. The theorem is readily proven when $p^{n}=3^{n}, n>1$; but for $p^{n}=3$ an additional generator is necessary and sufficient, viz.,

$$
W=\left(\begin{array}{llll}
1 & 2 & 2 & 2 \\
1 & 2 & 1 & 1 \\
1 & 1 & 2 & 1 \\
1 & 1 & 1 & 2
\end{array}\right), W^{3}=1
$$

$\dagger$ A preliminary account was presented before the Mathematical Conference at Chicago, December 30, 1897. 
are 2 and $\omega / 2$ for $m$ odd, and $2,2, \omega / 4$ for $m$ even. [For later results on the cases thus excluded see $\$ \$ 11-15$.

For $m$ odd, the orthogonal group $G$ had the same order and factors of composition as the linear Abelian group on $m-1$ indices. Judging from the results for the corresponding continuous groups of Lie, the resulting triply-infinite systems of simple groups of the same order $\omega / 2$ are probably isomorphic when $m=3$, but not when $m>3$. [See $\$ 14$.

Excluding here the cases $m=5,6,7$, which require lengthy special investigations, I will now give a short, simple proof of the above result. The complete memoir will appear in the Proceedings of the California Academy of Sciences.*

The substitutions $O_{1,2}^{\alpha, \beta}$ form a commutative group of or$\operatorname{der} \dagger p^{n} \pm 1$. A subgroup of index 2 is formed by the substitutions

Indeed,

$$
Q_{1,2}^{\alpha, \beta}: \begin{aligned}
& \xi_{1}^{\prime}=\left(\alpha^{2}-\beta^{2}\right) \xi_{1}-2 \alpha \beta \xi_{2}, \\
& \xi_{2}^{\prime}=2 \alpha \beta \xi_{1}+\left(\alpha^{2}-\beta^{2}\right) \xi_{2} .
\end{aligned}
$$

$$
Q_{1,2}^{\gamma, \delta} Q_{1,2}^{\alpha, \beta}=Q_{1,2}^{\alpha \gamma-\beta \delta, a \delta+\beta \gamma} \text {. }
$$

Further, its order is $\frac{1}{2}\left(p^{n} \pm 1\right)$ since $Q_{1,2}^{\alpha, \beta}$ and $Q_{1,2}^{\gamma, \delta}$ are identical if and only if $\alpha= \pm \gamma, \beta= \pm \delta$.

If $C_{i}$ denotes the substitution affecting only the index $\xi_{i}$, whose sign it changes, $C_{1} C_{2}$ is always contained among the substitutions $Q_{1,2}^{a, \beta}$.

Since we suppose that $p^{n}=8 l \pm 3,2$ is a not-square. Thus, with $\alpha^{2}+\beta^{2}=1$, we cannot have $\alpha^{2}-\beta^{2}=0$. Hence if $T_{12}$ denotes the transposition $\left(\xi_{1} \xi_{2}\right), T_{12} C_{1}$ is not of the form $Q_{1,2}^{\alpha, \beta}$ but serves to extend the group of the latter to the group of the $O_{1,2}^{a, \beta}$. Furthermore, if $j>2, T_{12} C_{1}$ transforms $Q_{1 j, j}^{\alpha \beta}$ into $Q_{2, j}^{a, \beta}$ and $Q_{2 j}^{\alpha, \beta}$ into $Q_{1, j}^{\alpha,-\beta}$. Hence if we extend the alternating group on the $m$ letters $\xi_{i}$ by the substitutions $Q_{1,2}^{\alpha, \beta}$ we obtain a group $H$ of index 2 under $G$.

3. Theorem: For $p^{n}>5, m>7$, the maximal invariant subgroup of $H$ is of order 2 or 1 according as $m$ is even or odd.

For $m$ even, $H$ contains an invariant subgroup of order 2 generated by the substitution

$$
N: \quad \xi_{i}^{\prime}=-\xi_{i} \quad(i=1, \cdots, m) .
$$

Suppose $H$ has an invariant subgroup $I$ containing a substitution

\footnotetext{
* Third Series, vol. 1, No. 4 ; the later results in No. 5.

+ "Orthogonal Group in a Galois Field," \& 3 ; Bullersin, February, 1898, pp. 196-200.
} 


$$
S: \quad \boldsymbol{\xi}_{i}^{\prime}=\sum_{j=1}^{m} a_{i j} \xi_{j} \quad(i=1, \cdots, m),
$$

neither the identity nor $N$. By suitably transforming $S$, we can suppose that $\alpha_{11}{ }^{2}+\alpha_{21}{ }^{2}+1$. Then $S$ is not commutative with $C_{1} C_{2}$; for, if so, it would be merely a product of a substitution affecting only $\xi_{1}$ and $\xi_{2}$ by a substitution affecting only $\xi_{3}, \cdots, \xi_{m^{*}}$. Hence the group $I$ contains the substitution, not the identity,

$$
S^{-1} C_{1} C_{2} S C_{1} C_{2}=S_{a} C_{1} C_{2}
$$

where $S_{a} \equiv S^{-1} C_{1} C_{2} S$, of period two, is found to be

$$
\xi_{i}^{\prime}=\xi_{i}-2 \alpha_{i 1} \sum_{j=1}^{m} \alpha_{j 1} \xi_{j}-2 \alpha_{i 2} \sum_{j=1}^{m} \alpha_{j 2} \xi_{j} \quad(i=1, \cdots, m) .
$$

4. Lemma: The orthogonal substitution

$$
\begin{aligned}
\xi_{i}^{\prime} & =\lambda \xi_{i}+\mu \xi_{j}+\nu \xi_{k}, \\
O_{i j k}: \quad & \xi_{j}^{\prime}=\lambda^{\prime} \xi_{i}+\mu^{\prime} \xi_{j}+\nu^{\prime} \xi_{k}, \\
\xi_{k}^{\prime} & =\lambda^{\prime \prime} \xi_{i}+\mu^{\prime \prime} \xi_{j}+\nu^{\prime \prime} \xi_{k},
\end{aligned}
$$

where $\lambda^{2}+\mu^{2}+\nu^{2}=1, \quad \lambda \lambda^{\prime}+\mu \mu^{\prime}+\nu \nu^{\prime}=0, \quad$ etc. transforms $S_{a}$ into $S_{a^{\prime}}$ where

$$
\begin{aligned}
& \alpha_{i l}^{\prime}=\lambda \alpha_{i l}+\mu \alpha_{j l}+\nu \alpha_{k l}, \\
& \alpha_{j l}^{\prime}=\lambda^{\prime} \alpha_{i l}+\mu^{\prime} \alpha_{j l}+\nu^{\prime} \alpha_{k l}, \\
& \alpha_{k l}^{\prime}=\lambda^{\prime \prime} \alpha_{i l}+\mu^{\prime \prime} \alpha_{j l}+\nu^{\prime \prime} \alpha_{k l}, \\
& \alpha_{s l}^{\prime}=\alpha_{s l}, \quad(s=1, \cdots, m, s+i, j, k) .
\end{aligned}
$$

If $\alpha_{i 1} \neq 0$, we can choose $\lambda, \mu, \nu$ such that $\alpha_{i 1}{ }^{\prime}=0$. For if $\alpha_{i 1}^{2}+\alpha_{j_{1}}^{2}=0$ and therefore $\alpha_{j 1} \neq 0$, we may take

$$
\lambda=\frac{-\alpha_{k 1}}{2 \alpha_{i 1}}, \quad \mu=\frac{-\alpha_{k 1}}{2 \alpha_{j 1}}, \quad \nu=1 \text {. }
$$

If $\alpha_{i 1}^{2}+\alpha_{j 1}^{2} \neq 0$, we derive the equivalent condition,

$$
\left\{\mu\left(\alpha_{i 1}^{2}+\alpha_{j 1}^{2}\right)+\nu \alpha_{j 1} \alpha_{k 1}\right\}^{2}+\nu^{2} \alpha_{i 1}^{2}\left(\alpha_{i 1}^{2}+\alpha_{j 1}^{2}+\alpha_{k 1}^{2}\right)=\alpha_{i 1}^{2}\left(\alpha_{i 1}^{2}+\alpha_{j 1}^{2}\right),
$$

which has solutions* for $\mu$ and $\nu$ in the $G F\left[p^{n}\right]$ except when $\alpha_{i 1}^{2}+\alpha_{j 1}^{2}+\alpha_{k 1}^{2}=0$. In the latter case the condition $\lambda^{2}+\mu^{2}$ $+\nu^{2}=1$ may be written

$$
\alpha_{i 1}^{2}=-\left(\mu \alpha_{k 1}-\nu \alpha_{j 1}\right)^{2},
$$

having solutions if and only if -1 be a square.

* Bulletin, l.c. \& 3. 
5. Lemma: $O_{i j k l}$ transforms $S_{a}$ into $S_{a^{\prime}}$, where

$$
\alpha_{i 1}^{\prime}=\lambda \alpha_{i 1}+\mu \alpha_{j 1}+\nu \alpha_{k 1}+\sigma \alpha_{l 1} .
$$

If $\alpha_{i 1}^{2}+\alpha_{j 1}^{2}+\alpha_{k 1}^{2}=0$, the values

$$
\lambda=\frac{-\alpha_{\imath 1}}{a_{i 1}}, \quad \mu=\frac{\alpha_{k 1} \alpha_{l 1}}{\alpha_{i 1}^{2}}, \quad \nu=\frac{-\alpha_{l 1} \alpha_{j 1}}{\alpha_{i 1}^{2}}, \quad \sigma=1
$$

make

$$
\alpha_{i 1}{ }^{\prime}=0, \quad \lambda^{2}+\mu^{2}+\nu^{2}+\sigma^{2}=1
$$

6. The invariant subgroup $I$ of $H$ was shown to contain the substitution $S^{\prime} \equiv S_{u} C_{1} C_{2}$ not the identity. Transforming $S^{\prime}$ successively by

$$
O_{i 345} \text { or } T_{12} C_{1} O_{i 345} \quad(i=m, m-1, \cdots, 6),
$$

according as the one or the other belongs to $H$, we obtain, by $\$ \$ 4-5$, a substitution $S_{\alpha^{\prime}} C_{1} C_{2}$ belonging to $I$ and having $\alpha_{m 1}{ }^{\prime}=\alpha_{m-1}{ }_{1}=\cdots=\alpha_{61}{ }^{\prime}=0$. Also, by $\S 4$, we may make $\alpha_{51}{ }^{\prime}$ $=0$; for we have

so that

$$
\alpha_{11}{ }^{2}+\alpha_{21}{ }^{2}=\alpha_{11}{ }^{2}+\alpha_{21}{ }^{2}+1,
$$

$$
\alpha_{51}{ }^{2}+\alpha_{41}{ }^{2}+\alpha_{31}{ }^{2}+0 \text {. }
$$

Next we transform $S_{a^{\prime}} C_{1} C_{2}$ successively by

$$
O_{j 567} \quad(j=m, m-1, \cdots, 8)
$$

and obtain in $I$ a substitution $S_{\alpha^{\prime \prime}} C_{1} C_{2}$ having

$$
\alpha_{j 1}{ }^{\prime \prime} \equiv \alpha_{j 1}^{\prime}=0, \quad \alpha_{j 2}^{\prime \prime}=0 \quad(j=m, \cdots, 8) .
$$

The group $I$ thus contains a substitution

$$
S: \quad \xi_{i}^{\prime}=\sum_{j=1}^{7} \beta_{i j j} \xi_{j} \quad(i=1, \cdots, 7)
$$

neither the identity nor $N \equiv C_{1} C_{2} \cdots C_{m}$.

7. If $S$ be commutative with every $C_{i}$, it is merely a product of an even number of the $C_{i}$, in which certain ones as $C_{k}$ are lacking. But if

$$
S=C_{i} C_{j} C_{r} C_{s} C_{t} \cdots,
$$

the group $I$ contains

$$
T_{i j} T_{i k} S T_{i k} T_{i j} S^{-1}=C_{k} C_{j}
$$

and hence, by transforming by suitable even substitutions, every product of two $C$ 's. But $H$ contains either $O_{1,2}^{\alpha, \beta}$ or $O_{1,2}^{a, \beta} T_{12} C_{1}$, which transform $C_{1} C_{3}$ into $Q_{1,2}^{a, \beta} C_{1} C_{3}$ and $Q_{1,2}^{a, \beta} C_{2} C_{3}$ 
respectively. Hence the group $I$ contains every $Q_{1,2}^{\alpha, \beta}$, among which, if $p^{n}>5$, occurs one different from the identity and from $C_{1} C_{2}$.

8. We may thus assume that $S$ is not commutative with $C_{1}$, for example. Supposing $m \equiv 8, S$ is commutative with $C_{8}$. Hence the group $I$ contains the substitution not the identity

$$
S^{-1} C_{1} C_{8} S C_{1} C_{8}=R_{\beta} C_{1}
$$

where $R_{\beta} \equiv S^{-1} C_{1} S$ is seen to be

$$
R_{\beta}: \quad \xi_{i}^{\prime}=\xi_{i}-2 \beta_{i 1} \sum_{i=1} \beta_{j 1}^{7} \xi_{j} \quad(i=1, \cdots, 7) .
$$

Transforming $R_{\beta} C_{1}$ by $O_{i 234}$ for $i=7,6,5$ successively, we may suppose that $\beta_{71}=\beta_{61}=\beta_{51}=0$.

It is readily seen that a substitution $R$ affecting only $\xi_{1}, \cdots, \xi_{4}$, is not commutative with every $T_{i j}(i, j=1, \cdots, 5)$ for example not with $T_{12}$. Then $I$ contains the substitution, not the identity,

$$
R^{-1} T_{12} T_{67} R T_{67} T_{12}=T_{\delta} T_{12},
$$

where $T_{\delta} \equiv R^{-1} T_{12} R$ has the form

where

$$
T_{\delta}: \quad \xi_{i}^{\prime}=\xi_{i}-\delta_{i} \sum_{j=1}^{4} \delta_{j} \xi_{j} \quad(i=1 \cdots 4),
$$

9. If $\delta_{3}=\delta_{4}=0, T_{\delta} T_{12}$ becomes $Q_{2,1}^{\alpha, \beta}$ if we set

$$
\alpha=\frac{1}{2}\left(\delta_{1}-\delta_{2}\right), \quad \beta=\frac{1}{2}\left(\delta_{1}+\delta_{2}\right) .
$$

Having $Q_{2,1}^{\alpha, \beta}, I$ contains also $Q_{2,3}^{\alpha, \beta}$ and $Q_{3,1}^{\alpha, \beta}$ and thus the product of the two, which reduces to $T_{\sigma} T_{12}$, having

$$
\sigma_{1}=-1, \quad \sigma_{2}=\alpha^{2}-\beta^{2}, \quad \sigma_{3}=2 \alpha \beta
$$

If $\alpha \cdot \beta=0, \quad Q_{2,1}^{a, \beta}=C_{1} C_{2}$, not being the identity. Then, by $\S 7, I$ contains every $Q_{i, j}^{\alpha, \beta}$ and therefore, if $p^{n}>5$, a substitution $Q_{2,3}^{a, \beta} Q_{3,1}^{\alpha, \beta}=T_{\sigma} T_{12}$ in which $\alpha \beta \neq 0$.

10. Thus $I$ contains a substitution $T_{\delta} T_{12}$ having $\delta_{3}$ and $\delta_{4}$ not both zero, say $\delta_{4} \neq 0$. Transforming it by $O_{346}$, we can make the resulting substitution $T_{\delta}^{\prime} T_{12}$ commutative with $T_{16}$. Indeed, the conditions

$$
\delta^{\prime}{ }_{6} \equiv \alpha \delta_{3}+\beta \delta_{4}=\delta_{1}, \quad \alpha^{2}+\beta^{2}+\gamma^{2}=1,
$$

combine into the single condition 


$$
\alpha^{2}\left(\delta_{3}^{2}+\delta_{4}^{2}\right)-2 \alpha \delta_{1} \delta_{3}+\delta_{4}^{2} \gamma^{2}=\delta_{4}^{2}-\delta_{1}^{2} .
$$

For $\delta_{3}^{2}+\delta_{4}^{2}=0$, a solution is given by $\gamma=0$ when $\delta_{1} \neq 0$, and by $\gamma=1$ when $\delta_{1}=0$. For $\delta_{3}^{2}+\delta_{4}^{2} \neq 0$, there exist* solutions $\alpha, \gamma$ in the $G F\left[p^{n}\right]$ of the equivalent equation of condition

$$
\left\{a\left(\delta_{3}^{2}+\delta_{4}^{2}\right)-\delta_{1} \delta_{3}\right\}^{2}+\delta_{4}^{2}\left(\delta_{3}^{2}+\delta_{4}^{2}\right) \gamma^{2}=\delta_{4}^{2}\left(\delta_{3}^{2}+\delta_{4}^{2}-\delta_{1}^{2}\right) .
$$

Hence $I$ contains the substitution, not the identity,

$$
\begin{gathered}
\left(T_{\delta^{\prime}} T_{12}\right)=T_{26} T_{78}\left(T_{\delta^{\prime}} T_{12}\right)^{-1} T_{78} T_{26} \\
=T_{\delta^{\prime}} T_{16} T_{\delta^{\prime}}^{-1} T_{26}=T_{16} T_{26^{\circ}}
\end{gathered}
$$

The alternating group on $m>4$ letters being simple, the group $I$, containing $T_{16} T_{26}$, contains the whole alternating group. Further, $C_{1} C_{2}^{16}$ transforms $T_{16} T_{26}$ into $T_{16} T_{26} C_{1} C^{9}$, so that $I$ contains $C_{1} C_{6}$ and therefore every $C_{i} C_{j}$. Hence, by $\$ 7, I$ contains every $Q_{1,2}^{a, \beta}$. Thus the group $I$ coincides with $H$.

Addenda $\dagger$ of April 18.

11. For $p^{n}=3$ or 5 , the maximal invariant sub-group of $H$ is of order 2 or 1 , according as $m>4$ is even or odd. For $p^{n}=3, m=4$, the order of $H$ is $2^{5} \cdot 3^{2}$, and its factors of composition are all primes.

12. Suppose $p^{n}=8 l \pm 1$, so that 2 is a square. Let $O_{1,2}^{a, \beta}$ denote a definite orthogonal substitution not in $Q_{1,2}$, so that $1 \pm \alpha$ are not-squares. Denote by $H_{1}$ the group obtained by extending the group of the $Q_{i, j}$ by all the products $O_{i, i}^{a, 9} O_{k, l}^{a, \beta}$.

TheORem : $H_{1}$ contains half of the substitutions of $G$. For every substitution of $G$ is of the form

$$
S=h_{1}()_{i, j}^{a, \beta} h_{2} O_{k, l}^{a, \beta} \cdots,
$$

$h_{1}, h_{2}, \cdots, h$ denoting substitutions of $H_{1}$. Now $O_{i, j}^{a, \beta}$ can be carried to the right of every $Q_{i, j}^{\lambda, \mu}$ and every $Q_{k, l}^{\lambda, \mu}(k, l \neq i, j)$. Further, since $\left(O_{1,2}^{\alpha, \beta}\right)^{2}=Q_{1,2}^{\alpha,-} \beta$,

$$
\begin{aligned}
& O_{i, j}^{\alpha, \beta} Q_{i, k}^{\lambda, \mu}=O_{i, j}^{\alpha, \beta}\left(O_{i, k}^{\alpha, \beta}\right)^{2} Q_{i, k}^{\alpha \beta} \cdot Q_{i, k}^{\lambda, \mu}, \\
= & O_{i, j}^{\alpha, \beta} O_{i, k}^{\alpha, \beta} \cdot Q_{i, k}^{\alpha, \beta} Q_{i, k}^{\lambda, \mu} \cdot O_{i, k}^{\alpha, \beta}=h O_{i, k}^{\alpha, \beta} .
\end{aligned}
$$

* Bulletin, 1. c. \& 3.

† The results here announced will be proven in full in the Proceedings of the California Academy of sciences, Third Series, vol. 1, No. 5. 
Thus $S$ finally takes the form

$$
h^{\prime} O_{r, s}^{\alpha, \beta}=h^{\prime} O_{r, s}^{\alpha, \beta} O_{2,1}^{\alpha, \beta} O_{1,2}^{\alpha, \beta}=h^{\prime \prime} O_{1,2}^{\alpha, \beta} .
$$

13. Theorem: For $m>4, p \neq 2$, the maximal invariant sub group of $H_{1}$ is of order 2 or 1 according as $m$ is even or odd.

For $m>7$, the group is similar to that for the group $H$ as given above. In $\$ 6$ we replace $T_{12} C_{1} O_{i 345}$ by $O_{1,2}^{\alpha, \beta} O_{i 345^{\circ}}$ We replace $\$ 7$ by the

Lemma: If $p^{n}=8 l \pm 1, m>3$, an invariant sub-group I containing every $C_{i} C_{j}$ coincides with $H_{1}$.

Indeed, $O_{2,4}^{\alpha, \beta} O_{1,2}^{\alpha, \beta}$ transforms $C_{1} C_{3}$ into $Q_{1,2}^{\alpha, \beta} C_{1} C_{3}$, so that $I$ contains every $Q_{i, j}^{\alpha \beta}$. Having $T_{23} C_{3}, I$ contains every $O_{i, j}^{a, \beta} \quad O_{k, l}^{a, \beta}$. Thus, for example,

$$
\left(T_{23} C_{3}\right)\left(\begin{array}{ll}
O_{1,2}^{\alpha, \beta} & O_{1,4}^{\alpha, \beta}
\end{array}\right)\left(T_{23} C_{3}\right)^{-1}\left(O_{1,2}^{\alpha, \beta} O_{1,4}^{\alpha, \beta}\right)^{-1}=O_{1,3}^{\alpha, \beta} O_{2,1}^{\alpha, \beta} .
$$

14. Theorem : For $p>2$, the ternary orthogonal group in the $G F\left[p^{n}\right]$ has a sub.group $H^{\prime}$ of index two and of order $\frac{1}{2} p^{n}\left(p^{2 n}-1\right)$ which is simply isomorphic to the group of linear fractional substitutions of determinant unity on a single index.

Indeed, the orthogonal substitution

$$
S: \xi_{i}^{\prime}=\sum_{j=1}^{3} \alpha_{i j} \xi_{j} \quad(i=1,2,3),
$$

expressed in terms of the new indices

$$
\eta_{1}=-i \xi_{1}, \quad \eta_{2}=\xi_{2}-i \xi_{3}, \quad \eta_{3}=\xi_{2}+i \xi_{3},
$$

leaves $\eta_{1}{ }^{2}-\eta_{2} \eta_{3}$ invariant and has the form

$$
S_{1}:\left\{\begin{array}{clc}
\alpha_{11} & \frac{1}{2}\left(\alpha_{13}-i \alpha_{12}\right) & -\frac{1}{2}\left(\alpha_{13}+i \alpha_{12}\right) \\
\alpha_{31}+i \alpha_{21} & \frac{1}{2}\left(\alpha_{22}-i \alpha_{32}+i \alpha_{23}+\alpha_{33}\right) & \frac{1}{2}\left(\alpha_{22}-i \alpha_{32}-i \alpha_{23}-\alpha_{33}\right) \\
-\alpha_{31}-i \alpha_{21} & \frac{1}{2}\left(\alpha_{22}+i \alpha_{32}+i \alpha_{23}-\alpha_{33}\right) & \frac{1}{2}\left(\alpha_{12}+i \alpha_{32}-i \alpha_{23}+\alpha_{33}\right)
\end{array}\right\}
$$

Understanding by $H^{\prime}$ the group $H$ or $H_{1}$ according as $p^{n}=8 l \pm 3$ or $p^{n}=8 l \pm 1$, we may verify that for every substitution of $H^{\prime}$ the coefficient $\frac{1}{2}\left(\alpha_{22}-i \alpha_{32}+i \alpha_{23}+\alpha_{33}\right)$ is the square of a complex $\alpha$ of the form $\rho+\sigma i$, where $\rho$ and $\sigma$ are marks of the $G F\left[p^{n}\right]$. It readily follows $*$ that $S_{1}$ may be written in the form :

$$
S_{1}:\left\{\begin{array}{rrr}
\alpha \delta+\beta \gamma & \alpha \gamma & \beta \delta \\
2 \alpha \beta & \alpha^{2} & \beta^{2} \\
2 \gamma \delta & \gamma^{2} & \delta^{2}
\end{array}\right\} \quad[\alpha \delta-\beta \gamma=1]
$$

where $\alpha$ is conjugate to $\delta, \beta$ to $\gamma$. Further two such ternary

* Compare Klein-Fricke : Automorphic Functions I., p. 14 ; Weber: Algebra, II., p. 190. 
substitutions have the same composition formula as linear fractional substitutions. Hence, according as -1 is a square or a not.square, $H^{\prime}$ is simply isomorphic to the "real" or the "imaginary" form * of the group of linear fractional substitutions of determinant unity. Thus, for $p^{n}>3, H^{\prime}$ is simple.

15. Observing that the squares of the substitutions

$$
O_{1,2}^{a, \beta}, \quad O_{1,2}^{a, \beta} T_{13} C_{1} C_{2} C_{3}, \quad O_{1,2}^{a, \beta} T_{13} T_{24}
$$

are respectively $Q_{1,2}^{\alpha,-\beta}, \quad O_{1,2}^{\alpha, \beta} O_{3,2}^{\alpha, \beta}, \quad O_{1,2}^{\alpha, \beta} O_{3,4}^{\alpha, \beta}$, we may unite our results into the following

TheOREM : The squares of the linear substitutions on $m$ indices in the $G F\left[p^{n}\right], p \neq 2$, which leave invariant the sum of the squares of the $m$ indices, generate a group, which for $m=2 k+1$ has the order

$$
\frac{1}{2}\left(p^{2 n k}-1\right) p^{2 n k-n}\left(p^{2 n k-2 n}-1\right) p^{2 n k-3 n} \cdots\left(p^{2 n}-1\right) p^{n}
$$

and is simple except when $p^{n}=3, m=3$; while for $m=2 k>4$ it has the factors of composition 2 and

$$
\frac{1}{4}\left[p^{n k}-( \pm 1)^{k}\right] p^{n k-n}\left(p^{2 n k-2 n}-1\right) p^{2 n k-3 n} \cdots\left(p^{2 n}-1\right) p^{n} \text {, }
$$

the sign \pm depending upon the form $4 l \pm 1$ of $p^{n}$.

UNIVERSITY OF CALIFORNIA,

February 10, 1898.

\section{A PROOF OF THE THEOREM :}

$$
\frac{\partial^{2} u}{\partial x \partial y}=\frac{\partial^{2} u}{\partial y \partial x}
$$

BY MR. J. K. WHITTEMORE.

(Read before the American Mathematical Society at the Meeting of April $30,1898$.

THEOREm : Let $u=f(x, y)$ denote a function of the two independent variables $x$ and $y$ which, together with its first derivatives and the two second derivatives in question, is continuous in the neighborhood of the point $(x, y)$; then $\frac{\partial^{2} u}{\partial x \partial y}=\frac{\partial^{2} u}{\partial y \partial x}$

$$
\text { Let } \frac{\partial^{2} f(x, y)}{\partial x \partial y} \text { denote } \frac{\partial}{\partial x}\left(\frac{\partial f(x, y)}{\partial y}\right)
$$

* Moore : Mathematical Papers of the Chicago Congress (1893), "A doubly-infinite system of simple groups," $\& \& 5-6$. 